\title{
Restoration of Sagittal Balance in Spinal Deformity Surgery
}

\author{
Melvin C. Makhni, M.D., M.B.A., Jamal N. Shillingford, M.D., Joseph L. Laratta, M.D., ${ }^{1}$ Seung-Jae Hyun, M.D., Ph.D., ${ }^{2}$ \\ Yongjung J. Kim, M.D.' \\ Department of Orthopaedic Surgery, ${ }^{\top}$ Spine Service, Columbia University College of Physicians and Surgeons, New York, NY, USA \\ Department of Neurosurgery, ${ }^{2}$ Spine Center, Seoul National University Bundang Hospital, Seoul National University College of Medicine, \\ Seongnam, Korea
}

The prevalence of patients with adult spinal deformity (ASD) has been reported as high as $68 \%$. ASD often leads to significant pain and disability. Recent emphasis has been placed on sagittal plane balance and restoring normal sagittal alignment with regards to the three dimensional deformity of ASD. Optimal sagittal alignment has been known to increase spinal biomechanical efficiency, reduce energy expenditure by maintaining a stable posture with improved load absorption, influence better bony union, and help to decelerate adjacent segment deterioration. Increasingly positive sagittal imbalance has been shown to correlate with poor functional outcome and poor self-image along with poor psychological function. Compensatory mechanisms attempt to maintain sagittal balance through pelvic rotation, alterations in lumbar lordosis as well as knee and ankle flexion at the cost of increased energy expenditure. Restoring normal spinopelvic alignment is paramount to the treatment of complex spinal deformity with sagittal imbalance. Posterior osteotomies including posterior column osteotomies, pedicle subtraction osteotomies, and posterior vertebral column resection, as well anterior column support are well known to improve sagittal alignment. Understanding of whole spinal alignment and dynamics of spinopelvic alignment is essential to restore sagittal balance while minimizing the risk of developing sagittal decompensation after surgical intervention.

Key Words : Sagittal plane · Spinal deformity · Alignment · Osteotomy.

\section{INTRODUCTION}

The prevalence of patients with adult spinal deformity (ASD) has been reported as high as $68 \%{ }^{50)}$. These patients with ASD often complain of significant pain and disability. Emphasis had traditionally been focused on improving coronal plane alignment. However, there has been recent literature stressing the importance of sagittal plane balance and the significance of restoring normal sagittal alignment with regard to the three dimensional deformity of ASD.

Optimal sagittal alignment improves spinal biomechanical efficiency, reduces energy expenditure and decelerates adjacent segment degeneration. Sagittal imbalance physiologically causes an increased reliance on accessory muscles to stay erect and therefore, increased work expenditure during gait ${ }^{45}$. The increased workload leads to early fatigue as well as pain especially in the back, buttocks, and thighs. Individuals with either flexible or rigid deformities may inherently attempt to com-

- Received : April 27, 2017 •Revised : June 7, 2017 •Accepted : June 13, 2017

- Address for reprints : Seung-Jae Hyun, M.D., Ph.D.

Department of Neurosurgery, Spine Center, Seoul National University Bundang Hospital, Seoul National University College of Medicine, 82 Gumi-ro 173beon-gil, Bundang-gu, Seongnam 13620, Korea

Tel : +82-31-787-7164, Fax : +82-31-787-4097, E-mail : hyunsj@snu.ac.kr

This is an Open Access article distributed under the terms of the Creative Commons Attribution Non-Commercial License (http://creativecommons.org/licenses/by-nc/4.0) which permits unrestricted non-commercial use, distribution, and reproduction in any medium, provided the original work is properly cited. 
pensate through pelvic retroversion, hip extension, or hip and knee flexion for severe fixed imbalance. These compensatory mechanisms further increase energy expenditure.

The purpose of this article is to define the various global and regional sagittal parameters with sagittal balance or imbalance, to understand the changes in various global and regional sagittal parameters as well as the Scoliosis research society (SRS)22 outcome scores with sagittal imbalance syndrome and after rebalancing surgery. Finally this article will discuss the various surgical options, including posterior column osteotomies (PCOs) and anterior structural grafts, utilized to achieve optimal outcomes.

\section{GLOBAL AND REGIONAL SAGITTAL PARAM- ETERS}

Various regional and global parameters help define "normal" sagittal alignment (Table 1). The $\mathrm{C} 7$ sagittal vertical axis (SVA) is determined by the C7 plumb line drawn caudally from the center of the body of $\mathrm{C}$. The line should theoretically intersect the posterosuperior aspect of the $\mathrm{S} 1$ endplate on a full-length standing X-ray. By definition, if the line passes anteriorly, the patient has positive global sagittal balance, and if the line passes posteriorly, the patient has negative balance. Typically, in patients with normal or neutral sagittal balance, the $\mathrm{C} 7$ plumb line should fall within $3 \mathrm{~cm}$, either anterior $(3$ $\mathrm{cm}$ positive) or posterior ( $3 \mathrm{~cm}$ negative), to the posterosuperior aspect of the S1 endplate. To obtain an even more global view of sagittal balance, it is possible to measure the balance

Table 1. Normal sagittal parameters : global and regional

\begin{tabular}{lc}
\hline Sagittal parameter & Average measurement \\
\hline C2-7 SVA & $15+11.2 \mathrm{~mm}$ \\
C7 SVA & $0+3 \mathrm{~cm}$ \\
C1-2 lordosis & $35^{\circ}\left(\right.$ range, 8 to $\left.55^{\circ}\right)$ \\
C2-7 lordosis & $13^{\circ}\left(\right.$ range, 0 to $\left.54^{\circ}\right)$ \\
T5-12 kyphosis & $30^{\circ}\left(\right.$ range, 20 to $\left.50^{\circ}\right)$ \\
T10-L2 & $0^{\circ}$ \\
T12-S1 & $60^{\circ}\left(\right.$ range, 31 to $\left.79^{\circ}\right)$ \\
Sacral slope & $40+9^{\circ}$ \\
Pelvic tilt & $11^{\circ}$ \\
\hline Pelvic incidence & $52+9^{\circ}$ \\
\hline
\end{tabular}

SVA : sagittal vertical axis between the basion to $\mathrm{S} 1$ or the basion to the center of the femoral heads as well; mean values in asymptomatic controls were noted to be $23.4 \pm 14.9$ and $-26 \pm 37.5 \mathrm{~mm}$, respectively ${ }^{26)}$.

The T1 pelvic angle (TPA) has been introduced as a new predictor and assessment tool for both pre-operative and postoperative sagittal balance ${ }^{44)}$. Some authors believe that the SVA fails to account for pelvic compensation when assessing sagittal balance, which is integral for corrective procedures. SVA measurements can be affected by both patient posture and Xray magnification. TPA accounts for the interaction between SVA and pelvic tilt (PT) or trunk inclination and pelvic retroversion ${ }^{48)}$. TPA is measured by drawing a line from the center of the $\mathrm{T} 1$ vertebral body to the femoral heads and a line from the femoral heads to the center of the $\mathrm{S} 1$ endplate. The measurement can be made on a prone or upright X-ray. In a retrospective review of patients with adult scoliosis undergoing corrective surgery with pedicle subtraction osteotomy (PSO), TPA was found to categorize spinal deformity in a manner frequently consistent with the SRS-Schwab Classification, and larger TPA values correlated with worse health outcome measures. The authors defined target TPA of 10-15 degree for patients aged 40-65 and 15-25 degree for patients older than 65 in order to decrease deterioration in patients with $\mathrm{ASD}^{48)}$.

Recently, age- and sex-based normative values of spinal alignment parameters were defined in a series of asymptomatic adult volunteers who underwent full-body radiographic imaging in order to provide deformity surgeons with a reference when planning corrective procedures ${ }^{26}$. Within the cohort of 115 volunteers without spine-related symptoms, it was noted that C7 SVA increased with age while lumbar lordosis (LL) decreased over time. Patients between 21-30 years old had average C7 SVA -27.1 mm, with gradual increase over each older decade until those over age 71 had C7 SVA of $28.2 \mathrm{~mm}$.

Regional measurements between vertebrae are evaluated from the cephalad endplate of the proximal level to the caudal end plate inferiorly. Cervical lordosis (CL) is measured between $\mathrm{C} 1-\mathrm{C} 2$ (average 35 degree, ranging from 8-55 degree) or between $\mathrm{C} 2-\mathrm{C} 7$ (average 13 degree, ranging from $0-54$ degree $)^{21,34,37,38)}$. Additionally, inherent relationships between cervical alignment and sagittal spinopelvic alignment have been reported. The $\mathrm{T} 1$ slope has been previously suggested as an important factor in influencing overall spinal sagittal alignment, and increasing $\mathrm{T} 1$ slope has been shown to significantly correlate with greater sagittal malalignment of the dens $\mathrm{s}^{1,21,34,37,38)}$. 
Another researcher found that C7 slope served as a valuable marker of overall sagittal plane alignment, acting as a link between the occipitocervical and thoracolumbar spine ${ }^{41)}$. The C7 slope is the angle created by a line parallel to the superior C7 endplate and a horizontal reference line. In their radiographic analysis, it was noted that $\mathrm{C} 7$ slope correlated significantly with both sacral slope (SS) and the C7 SVA. While the C7 plumb line between the center of $\mathrm{C} 7$ and the posterosuperior aspect of $\mathrm{S} 1$ describes the alignment below $\mathrm{C} 7$, recently there has been a growing emphasis on understanding balance above $\mathrm{C} 7$ as well. While the previously described C7 SVA can be used to define global balance, the C2 SVA can also be used (distance between the centroid of $\mathrm{C} 2$ or the odontoid and the posterosuperior aspect of the S1 endplate). To get a better understanding of the cervical alignment, the cervical SVA, of the distance between the centroid of $\mathrm{C} 2$ or the odontoid and the posterosuperior aspect of the C7 upper endplate (C2-C7 SVA), can also be used ${ }^{11}$. This parameter should be accounted for regularly since it correlates with multiple parameters including general health scores and severity of myelopathy; normal C2-C7 SVA is roughly $15-17 \pm 11.2 \mathrm{~mm}^{1,17)}$. Furthermore, a recent study shows that similar to the trend observed in the lumbosacral spine, T1 slope minus C2-C7 lordosis mismatch may significantly impact cervical alignment ${ }^{21)}$. Their results indicate that a greater mismatch between T1 slope and C2-C7 lordosis is associated with a greater degree of cervical malalignment and disability, as defined by C2-C7 SVA and neck disability index (NDI) scores (>25), respectively. Particularly, a T1S-CL mismatch greater than $22.2^{\circ}$ corresponded to severe disability (NDI $>25$ ) and positive cervical sagittal malalignment, defined as $\mathrm{C} 2-\mathrm{C} 7$ SVA greater than $43.5 \mathrm{~mm}$.

Thoracic kyphosis (TK) is measured from the upper endplate of T2 to the lower endplate of T12. Due to the radiographic shadow of the shoulders, a surrogate for TK can be estimated from the T5 upper endplate to the T12 lower endplate. Normal TK is estimated to be $20-50$ degree ${ }^{47}$. On average, the angle between horizontal reference line and upper endplate of T5 is 10 degree and that between the horizontal reference line and lower endplate of T12 is 20 degree. The thoracic spine has an average of 30 degree of kyphosis at T5-T12 (TK, described as “+”)".

Thoracolumbar (TL) kyphosis (normal 0 degree) is measured by the Cobb angle between the upper end-plate of T10 and lower end-plate of L2. Lumbar lordosis is the angle be- tween the lower endplate of the T12 vertebral body and the upper endplate of sacrum. It can also be measured between the cephalad endplate of $\mathrm{L} 1$ and the sacral endplate; normal values have been reported as 31-79 degree ${ }^{47)}$. Typically, the lumbar spine has 60 degree of lordosis from T12-S1 (LL, described as "-") "17). Over the last decade, however, the variability in LL has been appreciated, as well as its ideal correlation with the individual patient's pelvic incidence (PI) value. Up to two thirds of this lordosis routinely arises between $\mathrm{L} 4$ and $\mathrm{Sl}^{3}$.

SS describes the angle between the sacral endplate and the horizontal reference line, and is normally about 40 degree (normal described between $36-39 \pm 9$ degree ${ }^{511}$. PT describes the spatial orientation of the sacrum and the femoral head. PT is the angle between a vertical reference line and the line connecting the midpoint of the sacral end plate and the center of the femoral head, which is normally 11 degrees. PI describes the relationship of the sacrum to the pelvis and relates the spatial orientation of the femoral head to the sacrum; it is defined as the angle between a line perpendicular to the center of the sacral endplate and a line between the center of the sacral endplate and the center of the femoral heads. If the femoral heads are not aligned, the midpoint between the two femoral heads is used for measurement. Unlike other measures, PI is a fixed anatomic parameter that is not position-dependent; normal ranges have been described as about $52 \pm 9$ degree $^{39,51)}$. PI averages 52 degree in Caucasians, and slightly lower (47 degree) in patients of Asian descent ${ }^{3,36,39)}$. PI, PT, and SS share a unique relationship; PI equals the sum of PT and $\mathrm{SS}^{39)}$.

Spinal curvature parameters are inter-related and often proportional to each other. A small SS corresponds to a horizontal sacral endplate and decreased LL. On the other hand, patients with a more tilted sacral endplate and correspondingly increased SS have more pronounced LL. Similarly, TK and LL share a close relationship. Patients with a large TK are often found to have a large LL with increased slope at the T12 vertebral body. As discussed previously, PI is a static measurement with respect to positioning. Although it is a relatively fixed parameter, it has been noted to change with age. PI increases during childhood and adolescence and then remains relatively constant through adulthood. With the gradual loss of LL that occurs with aging, there is a further compensatory increase in PI as the pelvis rotates to maintain global spinal alignment ${ }^{16)}$. Above average PI $>60$ degree has been associated with high LL and found to be a predisposing factor in the development and pro- 
gression of spondylolisthesis secondary to alterations of mechanical stress at the lumbosacral junction.

Roussouly defined four types of patterns of sagittal alignment based on the location of the TL inflection point, or segment the TK transitions to $\mathrm{LL}^{47)}$. This change occurs at the L3/ L4 level in type 1 lordosis. Types 2-4 have progressively higher levels of transition at L1/L2, T12/L1, then T9/T10 for type 4. The PI increases as the inflection point lies more proximal and balance moves from negative to positive with type 3 being a well-balanced spine. Barrey et al. described patients in six groups of increasing PI values and found that LL increased in each group ${ }^{2)}$.

\section{SAGITTAL IMBALANCE AND COMPENSATORY MECHANISMS}

Sagittal imbalance may arise from a variety of causes. Congenital vertebral malformations or ankylosing spondylitis may prevent development of proper alignment, while tumors, infections, or degeneration may lead to progressive decompensation over time ${ }^{54)}$. Iatrogenic flatback syndrome results from surgical fusion with insufficient implementation of kyphosis or lordosis ${ }^{43)}$. Post-surgical changes, such as post-laminectomy kyphosis or adjacent segment disease at the ends of instrumented fusions, can also lead to sagittal imbalance through significant disc degeneration ${ }^{9,14,20)}$. Prior decompression, spinal extensor muscle and ligamentous damage from previous surgeries can also influence the development of sagittal deterioration. Patients with degenerative sagittal imbalance tend to be older with a history of prior distal lumbar spine surgery and often hypolordotic fusions ${ }^{5)}$.

Several compensatory mechanisms are employed in an effort to maintain neutral alignment in the sagittally malaligned patient. Overall, once there is a loss of lordosis by anterior column shortening, posterior lengthening, or increased kyphosis, the spine first attempts to compensate. Additional lordosis proximal and distal to the regionally imbalanced levels occurs, as well as a decrease in TK. As patients' age and their spines become increasingly rigid and kyphotic, the compensatory decrease in TK becomes exceedingly difficult. Then the pelvis is recruited and SS is decreased. Pelvic retroversion (corresponding to an increased PT) attempts to reposition the head over the pelvis and maintain a horizontal gaze. The erector muscles then help to correct posture but are unable to maintain the tension band effect due to degeneration and fatigue. If the prior compensatory mechanisms are not capable of maintaining sagittal balance, the lower extremities are recruited to alter alignment. If not sufficiently compensated through pelvic retroversion, hip flexion, knee flexion and even ankle flexion attempts to retain balance at the cost of increased energy expenditure $^{13)}$. Hip flexion contracture is possible with long lasting sagittal imbalance which sometimes makes sagittal rebalancing surgery more complicated. Hyperextending the occiput through C2 segments may be employed to maintain horizontal gaze $^{21,38)}$. Once there is a loss of balance of $5-8 \mathrm{~cm}$ positive imbalance, a vicious cycle proceeds in which further imbalance puts more stress on compensatory mechanisms and leads to further decompensation. Global and regional changes have been reported in patients with sagittal imbalance ${ }^{32)}$. Kim et al. ${ }^{30)}$ reported on various sagittal parameters in 100 patients with an average sagittal imbalance of $15.5 \mathrm{~cm}$ vs. 100 asymptomatic controls with SVA $-0.8 \mathrm{~cm}$ (Table 2). The 100 patients with sagittal imbalance had 45 degree less LL (15 degree vs. 60 degree in asymptomatic adults), 14 degree less SS (24 vs. 38 degree in asymptomatic adults), 10 degree more PT (23 degree vs. 13 degree in asymptomatic adults), and 6 degree less of TK at T5-12 (26 vs. 32 degree in asymptomatic adults). The total loss of 45 degree LL corresponded to a $16.3 \mathrm{~cm}$ SVA difference in patients with sagittal imbalance despite thoracic compensation by loss of 6 degree TK and pelvic compensation by loss of 14 degree sacral slope.

Booth et al subdivided patients with sagittal imbalance into two groups (Fig. 1). Type 1 deformity referred to patients with segmental hypolordosis or kyphosis of the lumbar spine at previously fused levels but maintenance of global balance (i.e.,

Table 2. Various changes in sagittal parameter with type $2 \mathrm{SI}$

\begin{tabular}{lcc} 
& NI $(\mathbf{n}=\mathbf{1 0 0})$ & SI $(\mathbf{n}=100)$ \\
\hline C7 SVA $(\mathrm{cm})$ & $-0.8 \pm 3.1$ & $15.5 \pm 5.5$ \\
TK $\left(\right.$ T5-T12) $\left(^{\circ}\right)$ & $32 \pm 11.9$ & $26 \pm 20.3$ \\
\hline LL $\left(\right.$ T12-S1) $\left(^{\circ}\right)$ & $-60 \pm 11.6$ & $-15 \pm 20.3$ \\
Sacral slope $\left(^{\circ}\right)$ & $38 \pm 7.5$ & $24 \pm 16.3$ \\
Pelvic tilt $\left(^{\circ}\right)$ & $13 \pm 10.1$ & $33 \pm 14.5$ \\
TK+LL+PI $\left(^{\circ}\right)$ & $23 \pm 13.7$ & $68 \pm 18.3$ \\
TK+LL $\left(^{\circ}\right)$ & $-28 \pm 11.8$ & $12 \pm 23$ \\
\hline
\end{tabular}

$\mathrm{SI}$ : sagittal imbalance, $\mathrm{NI}$ : non imbalance, SVA : sagittal vertical axis, TK: thoracic kyphosis, LL : lumbar lordosis, PI : pelvic incidence 
Sagittal spino-pelvic compensation

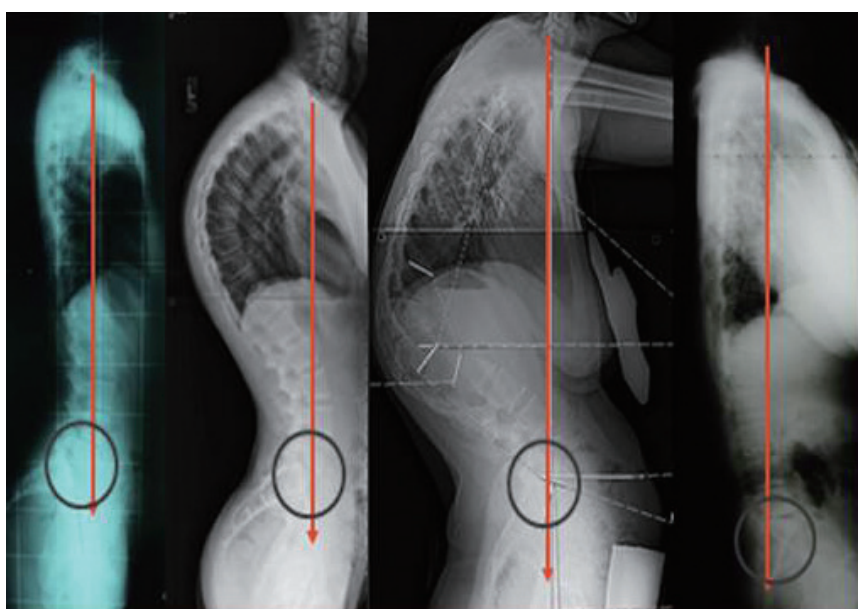

Type 1 : poor regional balance with good compensation (SVA<8 cm)
Sagittal spino-pelvic De-compensation

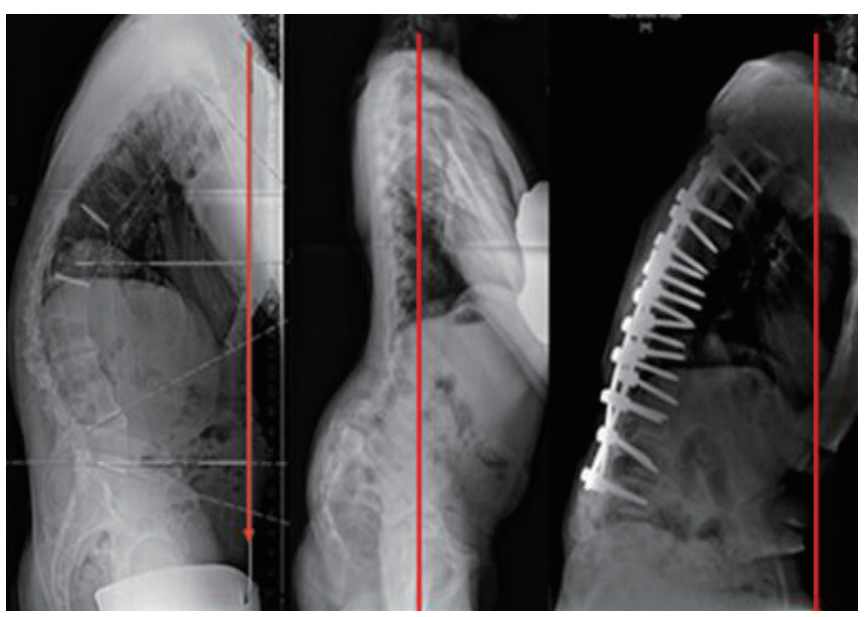

Type 2 : poor regional balance with poor compensation $(S V A>8 \mathrm{~cm})$

Fig. 1. Type 1 and 2 sagittal imbalance : examples of type 1 (compensated) and type 2 (decompensated) imbalance.

SVA $<8 \mathrm{~cm})^{45)}$. Type 2 global deformity was defined as having the $\mathrm{C} 7$ plumb line fall greater than $5 \mathrm{~cm}$ anterior to the lumbosacral disc with either fusion to the sacrum or significant disc degeneration below the fusion mass (i.e., SVA $>8 \mathrm{~cm}$ ). Type 1 patients are able to maintain sagittal balance by compensation through hyperextending mobile spine segments primarily below the fusion level, reflected through anterior disc heights $>5$ $\mathrm{mm}$ compared to posterior disc heights on radiograph. Knee flexion, pelvic retroversion (increase in pelvic tilt), and hip extension are several compensatory mechanisms utilized by patients with fixed sagittal imbalance to improve global alignment. Patients initially compensate for sagittal imbalance by standing with their feet spread further apart and knees flexed to improve the position of their head over their feet, attempting to optimize the $\mathrm{C} 7$ plumb $^{44)}$. Pelvic retroversion occurs next, which allows maximum hyperextension of the hips to maintain an optimal erect posture in the setting of loss of lordosis ${ }^{26}$. With ambulation, hip extensors quickly fatigue leading to a decompensated pitched forward posture. Unfused thoracic segments progressively develop a hypokyphotic posture to optimally compensate for a positive SVA and hypolordotic lumbar spine. Unfortunately, these compensatory mechanisms driven by dynamic changes in the unfused segments may predispose to accelerated disc degeneration ${ }^{26,44}$.

The Schwab adult deformity classification system, created in conjunction with the SRS, facilitates systematic organization spinal deformity and provides a guide for patient management. The classification system utilizes three sagittal plane modifiers and four curve type descriptors. The sagittal plane modifiers include PT $(0:<20$ degree, $+: 20$ to 30 degree, $++:>30$ degree), global alignment based on SVA $(0:<4 \mathrm{~cm},+: 4$ to 9.5 $\mathrm{cm},++:>9.5 \mathrm{~cm})$, and PI minus LL $(0:$ within 10 degree, + : moderate 10 to 20 degree, ++ : marked $>20$ degree). The spinopelvic parameters included in this classification system have been linked to pain and disability in deformity patients and may be used to determine thresholds for deformity correction. Optimization of these parameters significantly correlates with improvements in health-related quality of life measures post-operatively ${ }^{12)}$.

\section{RESTORATION OF SAGITTAL IMBALANCE}

Optimal sagittal balance is a distance from the $\mathrm{C} 7$ plumb to the posterosuperior endplate of $\mathrm{S} 1 \leq 3 \mathrm{~cm}$, and suboptimal as a distance of $3-8 \mathrm{~cm}$. In a retrospective analysis of factors controlling sagittal spinal balance following long adult instrumented TL fusion, the authors concluded that a sagittal Cobb angle difference of $>20$ degree between the LL and TK led to optimal sagittal balance in most cases. Optimal sagittal balance and the subsequent improved functional outcomes were found in patients with a smaller preoperative PI, smaller angular changes at the TL junction, and greater LL at final follow-up. Risk factors for suboptimal sagittal balancing included age older than 55 years at the time of surgery, postoperative PI compared to the difference between LL and TK of $>45$ de- 
gree, preoperative global sagittal balance $\geq 5 \mathrm{~cm}$, and smaller LL compared to $\mathrm{TK}<20$ degree postoperatively ${ }^{32)}$.

When planning a re-balancing surgery of the spine, it is difficult to predict how the spine will move above and below the osteotomy area. The amount of pelvic correction versus truncal correction from a given osteotomy must be estimated prior to surgery in order to properly and globally realign the deformed patient. In the evaluation of adult spinal deformity, the surgeon must first classify the key pathology as segmental, regional or global in order to guide surgical intervention. Further pre-operative assessment should include measurement of several elements integral to adequate correction. The SVA and T1 PT help to quantify the amount of sagittal imbalance. PI, TK, and LL should be evaluated in the context of PT. For example, patients with decreased LL compensate by pelvic retroversion and hip/knee flexion in order to maintain a horizontal gaze. The amount of lumbar deformity present can often be underestimated in situations of increased compensatory pelvic retroversion (PT). Pelvic retroversion (PT) is mainly affected by PI and LL. PI, pelvic tilt, and LL together determine the global sagittal balance (SVA). Therefore, by correcting the LL through operative intervention, the surgeon can modulate post-operative PT and restore global sagittal alignment (SVA).

The first step of sagittal re-balancing starts from adequate restoration of $\mathrm{LL}^{2,6)}$. The preferred method of the senior author is to correlate LL closely with PI despite many compensatory changes. The target LL is based on six classes of PI where patients with lower PI require less LL and patients with higher PI require greater LL in order to obtain optimal balance. In class I $\left(28^{\circ}<\mathrm{PI}<38^{\circ}\right)$, target $\mathrm{LL}$ is $\mathrm{PI}+18^{\circ}\left( \pm 7^{\circ}\right)$. In class II $\left(38^{\circ}\right.$ $\left.<\mathrm{PI}<48^{\circ}\right)$, target $\mathrm{LL}$ is $\mathrm{PI}+13^{\circ}\left( \pm 8^{\circ}\right)$. In class III $\left(48^{\circ}<\mathrm{PI}<58^{\circ}\right)$, target $\mathrm{LL}$ is $\mathrm{PI}+9^{\circ}\left( \pm 8^{\circ}\right)$. In class IV $\left(58^{\circ}<\mathrm{PI}<68^{\circ}\right)$, target $\mathrm{LL}$ is $\mathrm{PI}+6^{\circ}\left( \pm 5^{\circ}\right)$. In class $\mathrm{V}\left(68^{\circ}<\mathrm{PI}<78^{\circ}\right)$, target $\mathrm{LL}$ is $\mathrm{PI}+2^{\circ}\left( \pm 7^{\circ}\right)$. In class $\mathrm{VI}\left(78^{\circ}<\mathrm{PI}<88^{\circ}\right)$, target $\mathrm{LL}$ is $\mathrm{PI}-5^{\circ}\left( \pm 8^{\circ}\right)^{2)}$.

In a study of normal volunteers, Kim et al. ${ }^{30)}$ described relationship of PI, LL, and TK as PI+LL+TK=22, where LL is a negative value. Furthermore, the authors developed the formula PI+LL+TK<45 degree in order to guide surgical correction ${ }^{30,46)}$. At 91\% sensitivity, this formula has been proven successful at predicting ideal sagittal balance at 24 months postoperative$\mathrm{ly}^{30)}$. Kim et al. ${ }^{30)}$ further refined his formula, which details that the sum of TK+LL+PI should ideally lie between 20 and 45 degree for acceptable balance. This study also suggested correction of the TK to make optimal sagittal balance. Sometimes correction of thoracic hyperkyphosis (T5-T12 $\geq 50$ degree) or TL hyperkyphosis (T10-L2 $\geq 20$ degree) is needed to make optimal SVA postoperatively. Selection of fusion level to upper thoracic or TL spine should include the severity of global sagittal/coronal imbalance, thoracic or TL hyperkyphosisor osteoporosis or severe sagittal or coronal imbalance. When to stop at lower thoracic area, we should consider T10 is indeed the lowest immobile thoracic vertebra strut by the rib cage. Therefore we had better to decide to stop at T9 or T10 rather than T11 or T12 or L1 to prevent proximal junctional failure following sagittal plane corrective surgery.

Next step is to decide surgical approach to achieve the target LL. If the spine is flexible, lumbar extension positioning in the operation room may increase LL. Harimaya et al. ${ }^{18)}$ showed two different groups : those with increased LL ( $>5$ degree) during intraoperative prone positioning vs. those with minimal to no change in lordosis ( $<$ or $=5$ degree) during intraoperative prone positioning. The corresponding LL measurements for the increased lordosis group were -25.9 degree (upright), -40.0 degree (supine), -43.1 degree (intraoperative prone), and -48.9 degree (postoperative upright) ( $p<0.05$ for preoperative upright to all other comparisons). The corresponding LL measurements for the unchanged lordosis group were -54.2 degree, -53.8 degree, -50.3 degree, and -55.7 degree (no significant differences) ${ }^{18)}$. If the spine is not flexible by flexion/extension radiographs, patients require more aggressive posterior only corrective osteotomies or anterior structural grafts with or without posterior release to obtain satisfactory sagittal balance $^{6,12,33)}$. In case of long fusion to upper thoracic or lower thoracic spine, same strategic approach is recommended.

Anterior column structural grafts, either through a traditional anterior approach or a minimally invasive lateral approach, provide anterior column lengthening while precluding the wide exposure needed for posterior-based osteotomies. Because $60 \%$ to $80 \%$ of anatomic LL is found between L4 to S1, improved outcomes are observed if the majority of deformity correction and created LL is derived from these lower lumbar segments ${ }^{55}$. Complete release of the anterior longitudinal ligament (ALL) is inherent in these procedures and allows for maximal lordosis correction. Moreover, hyperlordotic cages (HLC) have been recently designed to further increase the correction ability. Twenty degree HLC can achieve up to 19 degree of segmental lordosis, while thirty degree HLC can achieve up to 29 degree of segmental lordosis with an average blood loss of only 240 
$\mathrm{mL}^{49)}$. Laterally based minimally invasive approaches can create 10.2 degree of segmental lordosis with operative times less than one hour and blood loss less than $50 \mathrm{~mL}^{11)}$. With similar sagittal correction magnitude to the PSO, the anterior or lateral procedures involving ALL release and anterior column structural support are a predictable method to correct LL and restore sagittal balance while mitigating the morbidity of the posterior dissection.

There are three general categories of osteotomies : PCOs, pedicle subtraction osteotomies, and vertebral column resections (VCR). Selecting the correct type of osteotomy in adult deformity is determined on a case-by-case basis and largely individualized to the specific patient. The decision of osteotomy or combination of osteotomies depends on a number of factors including the type, magnitude, stiffness, and angularity of the deformity as well as bone density, operative goals, and surgeon experience.

By definition, PCOs involve removal of the posterior ligaments and complete facetectomy, encompassing the classic Smith-Petersen and Ponte osteotomies. The amount of sagittal correction obtained from a PCO ranges from 5 to 10 degree per level, depending on the location of the osteotomy and flexibility of disc space. PCOs are the most commonly utilized osteotomies for the flexible spinal deformity patients, those with long smooth curves, those who need lesser amount of correction and those who are given anterior structural support. The relatively benign osteotomies do not carry the neurologic and pseudarthrosis risk of the more aggressive osteotomies.

Three column osteotomies are reserved for fixed, angular deformities. PSO is considered in patients with greater sagittal imbalance with fixed angular deformity. PSO involves removal of the posterior ligaments and facets with a transpedicular decancellation of the vertebral body allowing for simultaneous correction of coronal and sagittal plane deformities. Several studies demonstrated a single PSO can generate 20 to 40 degree of LL and an approximate 10 to $12 \mathrm{~cm}$ change in SVA, depending on the wedge of bone removed (Table 3$)^{6,10,23,25,31 \text { ) }}$. Unfortunately, despite aggressive correction with PSO, sagittal decompensation at ultimate follow-up (SVA $>8 \mathrm{~cm}$ ) may develop postoperatively in a small subset of patients (29\%). In these patients, sagittal decompensation is associated with significantly lower total SRS outcome scores and self-image and function subscale scores ${ }^{29}$. The presence of comorbidities, old age $>55$ years, preoperative higher $S V A>15 \mathrm{~cm}$, poor sagittal
Table 3. Various changes after lumbar PSO in SI patients

\begin{tabular}{lcc} 
& SI $(\mathbf{n}=\mathbf{1 1 4})$ & After PSO \\
\hline C7 SVA $(\mathrm{cm})$ & $14.2 \pm 6.03$ & $2.6 \pm 5.17$ \\
TK $\left(\right.$ T5-T12) $\left(^{\circ}\right)$ & $27 \pm 19.2$ & $35 \pm 15.1$ \\
\hline LL (T12-S1) $\left(^{\circ}\right)$ & $-15 \pm 20.3$ & $-52 \pm 14.5$ \\
\hline Sacral slope $\left(^{\circ}\right)$ & $23 \pm 15.8$ & $34 \pm 10.5$ \\
\hline Pelvic tilt $\left(^{\circ}\right)$ & $32 \pm 10.7$ & $21 \pm 9.1$ \\
TK+LL+PI $\left(^{\circ}\right)$ & $67 \pm 18.3$ & $38 \pm 15.3$ \\
\hline TK+LL $\left(^{\circ}\right)$ & $12 \pm 23$ & $-17 \pm 23$ \\
\hline
\end{tabular}

PSO : pedicle subtraction osteotomy, SI : sagittal imbalance, SVA : sagittal vertical axis, TK : thoracic kyphosis, LL : lumbar lordosis, PI : pelvic incidence

balance in 8 weeks postoperative SVA $>8 \mathrm{~cm}$, upper instrumented vertebra below $\mathrm{T} 8$, less LL angle increase $<40^{\circ}$, postoperative difference between $\mathrm{LL}$ and $\mathrm{TK}<25^{\circ}$, and TK+PI+LL (minus value) $<45^{\circ}$ are risk factors for developing postoperative sagittal decompensation ${ }^{28,46}$. There are several variants PSOs including asymmetrical pedicle osteotomy or discectomy. Hyun and colleagues reported fish-mouth PSO can provide a larger magnitude of correction comparing to classic PSO without compromising spinal cord function for fixed sagittal plane deformity ${ }^{42}$. They suggest that the ideal candidates for fish-mouth PSO are patients with fixed sagittal imbalance requiring a large magnitude of correction more than $35^{\circ}$ after 1-level osteotomy and the patients with angular kyphosis which apex has wedge deformity of the vertebral body.

VCR is a procedure of last resort and only considered when more conservative osteotomy will not suffice. Posterior VCR (PVCR) involves resection of all posterior elements, facet joints above/below, pedicles, entire vertebral body and discs above/below. VCR allows for tremendous ability to correct deformity as the entire spine is disarticulated and shortened. One PVCR corrected the sagittal curve by $40-80^{\circ}$ of kyphosis correction at lumbosacral and TL deformity. For sharp, angular kyphosis greater than $90^{\circ}$, posterior-only multilevel modified VCR is an effective way to correct the deformity ${ }^{56)}$. Unfortunately, the complication rate exceeded $30 \%$ including high degree of morbidity and significant blood loss ${ }^{53)}$. While three column osteotomies carry several risks such as for substantial blood loss, pseudarthrosis and neurologic compromise, they may be employed alone or in combination to achieve substantial sagittal as well as multiplanar correction ${ }^{6,12,33)}$. Pelvic fixation and multiple rods should be considered if upper instrumented vertebra is L2 or above and 3-column osteotomy is utilized. 
The use of a multiple-rod construct is a simple and effective method to provide increased stability across 3-column osteot- omy sites to significantly prevent implant failure and symptomatic pseudarthrosis vs. a standard 2-rod construct ${ }^{15,24)}$. Howev-
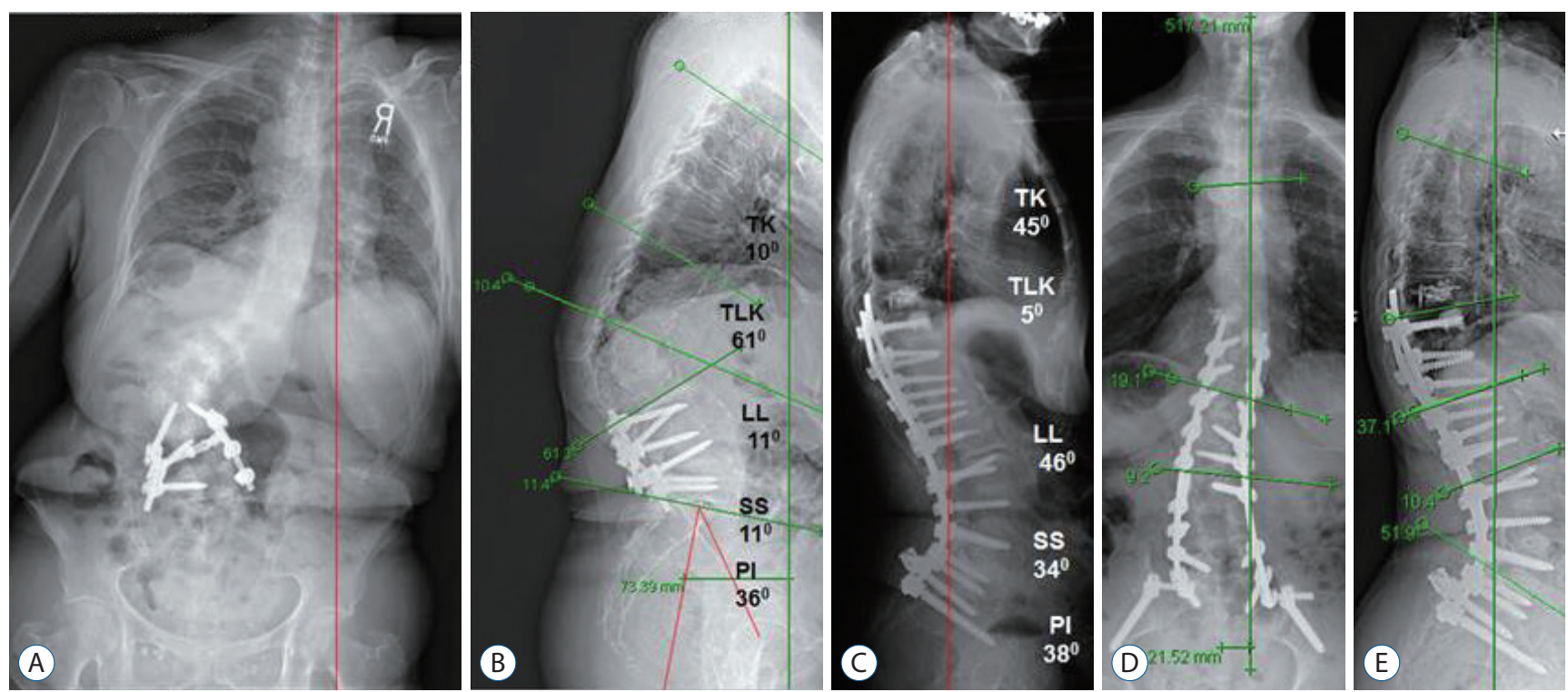

Fig. 2. A 72-year-old female with back pain and poor posture. Scoliosis AP radiographs (A) and lateral radiographs (B) reveal with poor balance and native $\mathrm{PI}$ of $36^{\circ}$. Target lumbar lordosis was $54^{\circ} \pm 7^{\circ}\left(47^{\circ}\right.$ to $\left.61^{\circ}\right)$. Mismatch between target $\mathrm{LL}$ and current $\mathrm{LL}$ was $34^{\circ} \pm 7^{\circ}\left(27^{\circ}\right.$ to $\left.41^{\circ}\right)$. Operative plan was a single PSO to obtain $35^{\circ}$ versus six posterior column osteotomies (PCOs) to obtain $30^{\circ}-42^{\circ}$. The decision was made to proceed with PSSIF T10-S1-Pelvis with PLIF at L5-S1 and PCOs at T10-11, T11-12, T12-L1, L1-2, L2-3, and L5-S1. Scoliosis lateral at 8 weeks (C) shows TK of $45^{\circ}$ and LL of $46^{\circ}$. Perfect sagittal balance was achieved. Scoliosis AP (D) and lateral (E) in 2 years. Perfect sagittal balance was maintained. TK : thoracic kyphosis, TLK : thoracolumbar kyphosis, LL : lumbar lordosis, SS : sacral slope, PI : pelvic incidence, AP : anterior posterior, PSO : pedicle subtraction osteotomy, PSSIF : posterior spinal segmental instrumentation and fusion, PLIF : posterior lumbar interbody fusion.
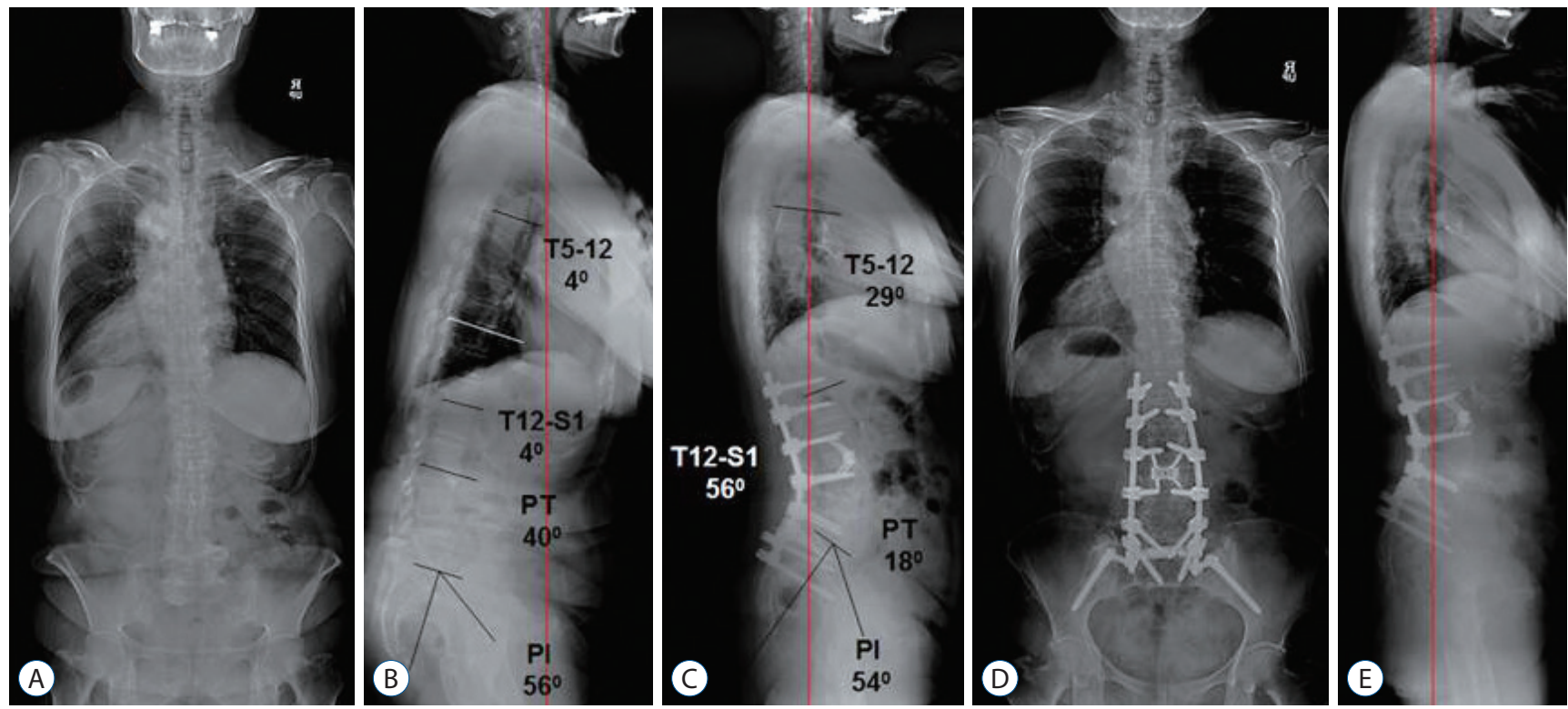

Fig. 3. A 60-year-old female with back pain and poor posture. Scoliosis AP radiographs (A) and lateral radiographs (B) reveal with poor balance and native PI of $56^{\circ}$. Target lumbar lordosis was $45^{\circ} \pm 8^{\circ}\left(37^{\circ}\right.$ to $\left.53^{\circ}\right)$. Mismatch between target $L L$ and current $L L$ was $61^{\circ} \pm 8^{\circ}\left(53^{\circ}\right.$ to $\left.69^{\circ}\right)$. Operative plan was a single pedicle subtraction osteotomy (PSO) $\left(35^{\circ}\right)$, multilevel posterior column osteotomies (PCOs) with or without anterior spinal fusion (ASF) (18 to $34^{\circ}$ ), and ASF at L2 to S1 $\left(20^{\circ}\right)$. The decision was made to proceed with PSSIF T12-S1-Pelvis with L4 PSO and PCO at L1-2, L4-5, and L5-S1. Scoliosis lateral at 8 weeks (C) shows TK of $29^{\circ}$ and $\mathrm{LL}$ of $56^{\circ}$. Perfect sagittal balance was achieved. Scoliosis AP (D) and lateral (E) at 4 years. Perfect sagittal balance was maintained. PT : pelvic tilt, PI : pelvic incidence, AP : anterior posterior, LL : lumbar lordosis, PSSIF : posterior spinal segmental instrumentation and fusion, TK: thoracic kyphosis. 

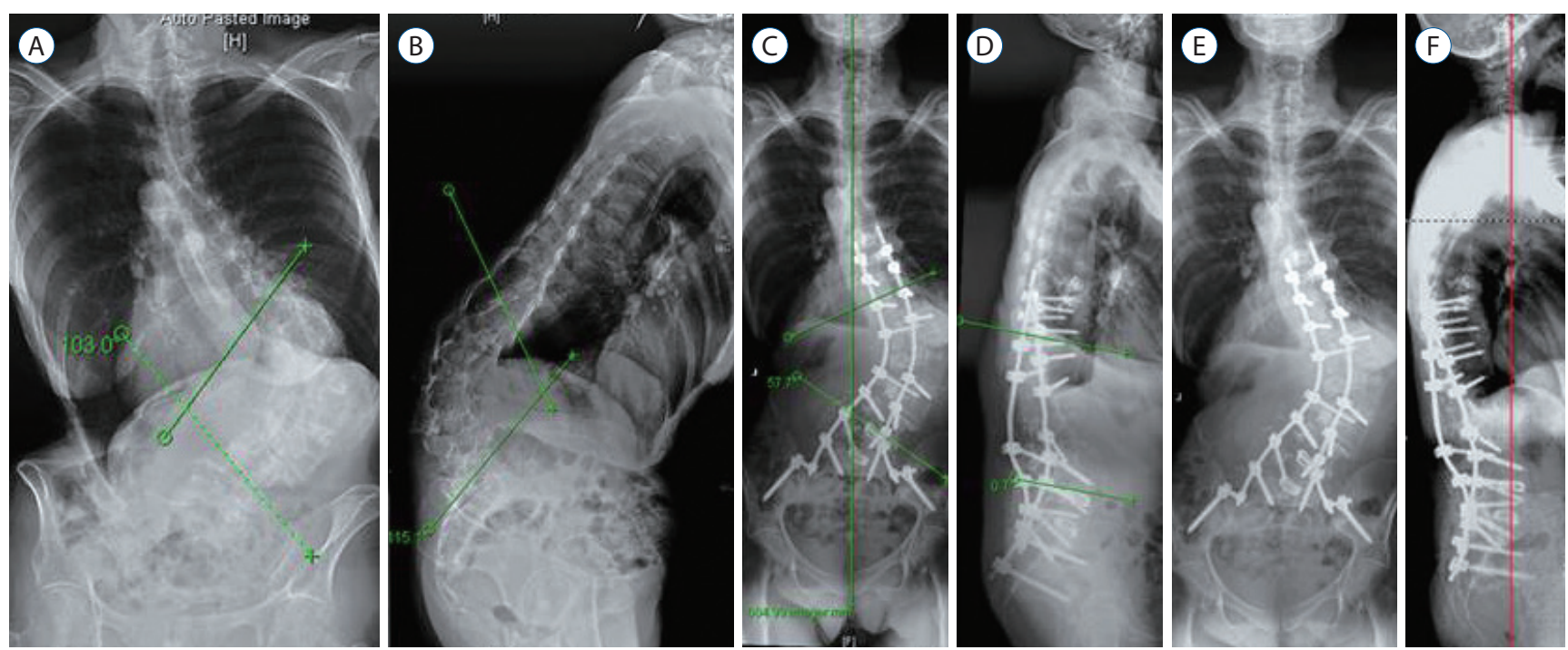

Fig. 4. A 73-year-old female with back pain and poor posture. Scoliosis AP radiographs (A) and lateral radiographs (B) reveal with massive fixed kyphoscoliosis of $103^{\circ}$ scoliosis and $115^{\circ}$ kyphosis. Operative plan was PSSIF T9-S1-Pelvis with L1 PVCR and PLIF at L4-5 and L5-S1 with multilevel PCOs. Scoliosis AP (C) and lateral (D) at 8 weeks show correction of scoliosis to $47^{\circ}$ and correction of kyphosis to $114^{\circ}$. Scoliosis AP (E) and lateral (F) at 4 years. Perfect sagittal balance was maintained. AP : anterior posterior, PSSIF : posterior spinal segmental instrumentation and fusion, PVCR : posterior vertebral column resections, PLIF : posterior lumbar interbody fusion, PCOs : posterior column osteotomies.

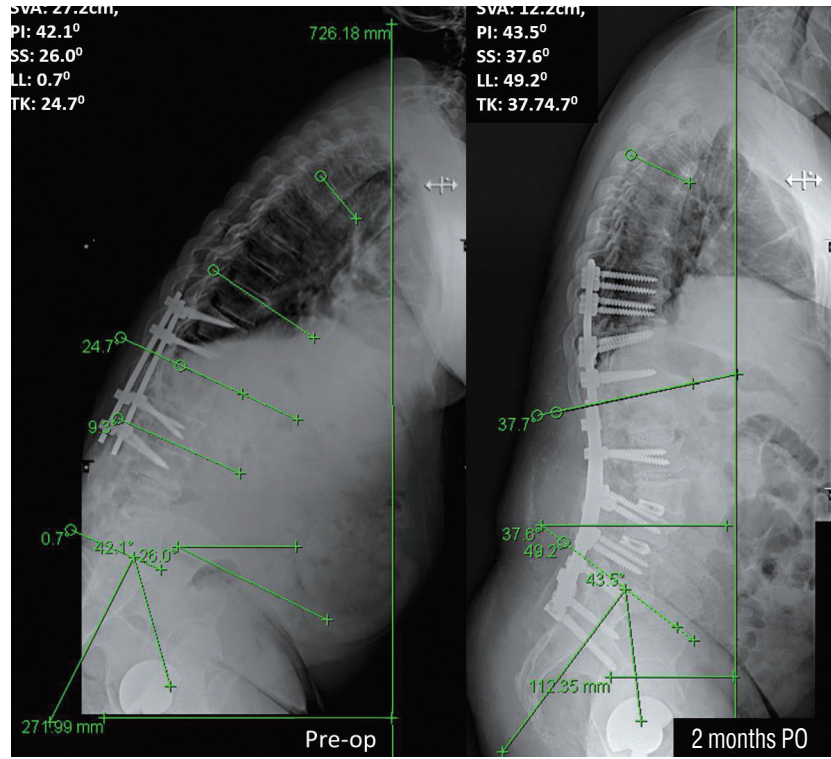

Fig. 5. A 61-year-old male who has severe sagittal imbalance of $27 \mathrm{~cm}$ and fixed hip contracture of 20 degree despite prior spinal instrumentation and fusion surgeries. Revision PSSIF at T9-Pelvis with 2 PLIFs at L3-4/ L4 -5 and PSO at L3 showed good restoration of lumbar lordosis from 0.70 to 49.20 . His preoperative PI was 42 degree. However, his SVA in 2 months is $12.2 \mathrm{~cm}$. Increase in thoracic kyphosis of 130 and sacral slope of 140 took out the advantage of good restoration of lumbar lordosis of 480 . Better sagittal vertical axis is expected in the future if flexion contracture of the hip is relieved with physical therapy. SVA : sagittal vertical axis, PI : pelvic incidence, SS : sacral slope, LL : lumbar lordosis, TK : thoracic kyphosis, PO : postoperative, PSSIF : posterior spinal segmental instrumentation and fusion, PLIF : posterior lumbar interbody fusion, PSO : pedicle subtraction osteotomy. er, a recent study shows that increased rod stiffness by use of Cobalt Chrome and multiple-rod construct can increase the incidence of proximal junctional kyphosis ${ }^{15)}$. Five illustrative cases are provided in Figs. 2-6.

\section{SAGITTAL BALANCE AND PATIENT OUTCOMES AFTER SAGITTAL RESTORATION}

Despite adequate correction, several studies have reported secondary loss of sagittal alignment postoperatively. Blondel et al. correlated low PI and lack of sufficient TK to postoperative loss of sagittal alignment regardless of surgical approach ${ }^{4,8}$. In a study on lumbar PSO, deterioration of unfused junctions occurred at a higher rate in older patients and those with worse preoperative PT, PI, or T1 spinopelvic inclination ${ }^{35}$. Similarly, Schwab and colleagues correlated postoperative loss of alignment with higher preoperative measurements of SVA, PT, and $\mathrm{PI}$, as well as LL-PI mismatch ${ }^{52}$.

Poor outcomes have been previously reported in patients with reduced LL and a fixed sagittal imbalance after spinal fusion. Several studies have elucidated the importance of sagittal balance and the correlation of positive imbalance with negative outcome scores ${ }^{13,19,40)}$. Moreover, a recent study showed a direct relationship between sagittal balance and quality of life scores ${ }^{40)}$. 


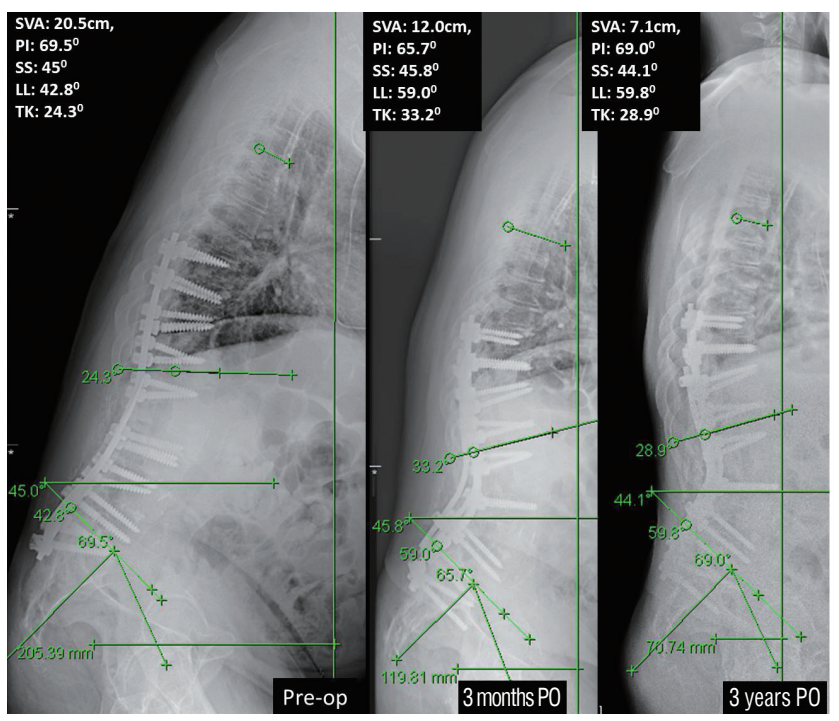

Fig. 6. A 52-year-old male who has severe sagittal imbalance of $21 \mathrm{~cm}$ and fixed hip contracture of 20 degree despite prior spinal instrumentation and fusion surgeries. Revision PSSIF at T9-Pelvis with 2nd PSO at L4 showed good restoration of lumbar lordosis from 42.80 to 59.00 . His preoperative PI was 69.50. However, his SVA in 3 months is $12.0 \mathrm{~cm}$. Increase in thoracic kyphosis of 90 and flexion contracture took out the advantage of restoration of lumbar lordosis. Significant improvement in sagittal vertical axis in 3 years to $7.1 \mathrm{~cm}$ came after relief of hip flexion contracture with physical therapy. SVA : sagittal vertical axis, $\mathrm{PI}$ : pelvic incidence, SS : sacral slope, LL : lumbar lordosis, TK : thoracic kyphosis, PO : postoperative, PSSIF : posterior spinal segmental instrumentation and fusion, PSO : pedicle subtraction osteotomy, PLIF : posterior lumbar interbody fusion.

Glassman and colleagues were the first to report on the detrimental pain and functional effects of sagittal imbalance greater than $4 \mathrm{~cm}^{13)}$. Likewise, Mac-Thiong et al. ${ }^{40)}$ attributed positive imbalance more than $6 \mathrm{~cm}$ to worse health related quality of life scores as evidenced by Oswestry Disability Index results. Self-image is equally affected. Positive sagittal balance has been associated with significantly worse self-image and social function, in addition to worse pain and poorer function ${ }^{13)}$. Fortunately, surgical correction of the imbalance with various osteotomies can lead to an over $90 \%$ improvement in self-image postoperatively ${ }^{7}$.

\section{SEVERAL ISSUES AFTER SAGITTAL REBAL- ANCING}

Normal population study showed that the distribution of ideal LL is between 10 degree less than PI and 10 degree more than PI in general. There is 20 degree difference in the distri-

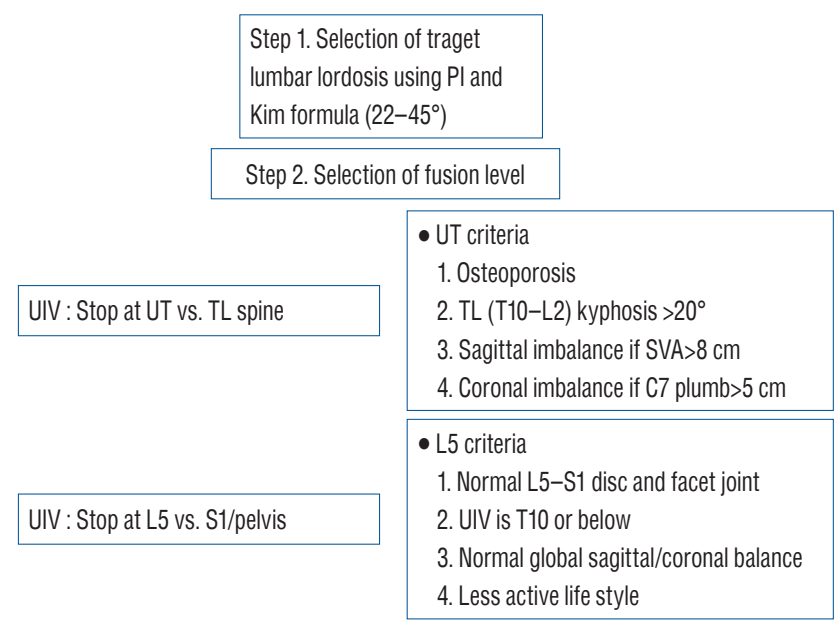

\begin{tabular}{|l|}
\hline $\begin{array}{c}\text { Step 3. Selection of surgical } \\
\text { approach (P vs. AP vs. PAP) }\end{array}$ \\
\hline Step 4 . Selection of osteotomies \\
PCO $: 5-10$ degree/level \\
PSO $: 20-40$ degree/level \\
PVCR $:>50$ degree
\end{tabular}

Fig. 7. Steps of sagittal re-balancing. PI : pelvic incidence, UIV : upmost instrumeted vertebra, UT : upper thoracic, TL : thoracolumbar, SVA : sagittal vertical axis, LIV : lower instrumeted vertebra, $\mathrm{P}$ : posterior, PA : posterior anterior, PAP : posterior anterior and posterior, PCO : posterior column osteotomie, PSO : pedicle subtraction osteotomy, PVCR : posterior vertebral column resections.

bution of ideal LL for given patient ${ }^{28-30,51,52)}$. Thus, we should make the target LL considering their age, bone quality, life style as well as PI. Previous studies show that reciprocal sagittal plane angle changes are variable such as SS or pelvic tilt and TK changes above the instrumented fusion area after LL restoration $^{29,47,52)}$. Stop at proximal thoracic spine rather than at the TL junction has advantage to make better SVA despite the loss of motion segment. The strategy should be followed especially in patients with osteoporosis, larger TK, paraspinal muscle atrophy and fatty degeneration at the TL junction area because symptomatic proximal junctional failure can occur more frequently (Fig. 7) ${ }^{15,22,27,28)}$. Lastly, flexion contracture of the hip joint needs more understanding.

\section{CONCLUSION}

Adult spinal deformity with sagittal imbalance is associated with significant pain, disability, as well as directly and negatively influence health related quality of life scores. The spine sur- 
geon has to understand the whole global and regional alignment changes after sagittal imbalance to address the multiplanar deformity. Restoration of global alignment and minimization of complications through various surgical options can successfully improve the pain and function of spinal deformity patients.

\section{CONFLICTS OF INTEREST}

The authors have no financial conflicts of interest.

\section{INFORMED CONSENT}

Informed consent was obtained from all individual participants included in this study.

\section{- Acknowledgements}

The submitted manuscript was presented by Yongjung J. Kim, M.D. as an invited speaker at the annual autumn meeting of The Korean Neurosurgical Society, 2015.

\section{References}

1. Ames CP, Blondel B, Scheer JK, Schwab FJ, Le Huec JC, Massicotte EM, et al. : Cervical radiographical alignment: comprehensive assessment techniques and potential importance in cervical myelopathy. Spine (Phila Pa 1976) 38(22 Suppl 1) : S149-S160, 2013

2. Barrey C, Jund J, Noseda O, Roussouly P : Sagittal balance of the pelvisspine complex and lumbar degenerative diseases. A comparative study about 85 cases. Eur Spine J 16 : 1459-1467, 2007

3. Bernhardt $\mathrm{M}$, Bridwell $\mathrm{KH}$ : Segmental analysis of the sagittal plane alignment of the normal thoracic and lumbar spines and thoracolumbar junction. Spine (Phila Pa 1976) 14 : 717-721, 1989

4. Blondel B, Schwab F, Bess S, Ames C, Mummaneni PV, Hart R, et al. : Posterior global malalignment after osteotomy for sagittal plane deformity: it happens and here is why. Spine (Phila Pa 1976) 38 : E394-E401, 2013

5. Bridwell $\mathrm{KH}$ : Causes of sagittal spinal imbalance and assessment of the extent of needed correction. Instr Course Lect 55 : 567-575, 2006

6. Bridwell KH : Decision making regarding Smith-Petersen vs. pedicle subtraction osteotomy vs. vertebral column resection for spinal deformity. Spine (Phila Pa 1976) 31(19 Suppl) : S171-178, 2006

7. Bridwell $K H$, Lewis SJ, Edwards C, Lenke LG, Iffrig TM, Berra A, et al. : Complications and outcomes of pedicle subtraction osteotomies for fixed sagittal imbalance. Spine (Phila Pa 1976) 28 : 2093-2101, 2003

8. Burkett B, Ricart-Hoffiz PA, Schwab F, Ialenti M, Farcy JP, Lonner BS, et al. : Comparative analysis of surgical approaches and osteotomies for the correction of sagittal plane spinal deformity in adults. Spine (Phila Pa 1976) 38 : 188-194, 2013

9. Cho KJ, Suk SI, Park SR, Kim JH, Jung JH : Selection of proximal fusion level for adult degenerative lumbar scoliosis. Eur Spine J 22 : 394-401, 2013

10. Choi HY, Hyun SJ, Kim KJ, Jahng TA, Kim HJ : Surgical and radiographic outcomes after pedicle subtraction osteotomy according to surgeon's experience. Spine (Phila Pa 1976) 24 : E795-E801, 2016

11. Deukmedjian AR, Le TV, Baaj AA, Dakwar E, Smith DA, Uribe JS : Anterior longitudinal ligament release using the minimally invasive lateral retroperitoneal transpsoas approach: a cadaveric feasibility study and report of 4 clinical cases. J Neurosurg Spine 17 : 530-539, 2012

12. Gill JB, Levin $A$, Burd $T$, Longley $M:$ Corrective osteotomies in spine surgery. J Bone Joint Surg Am 90 : 2509-2520, 2008

13. Glassman SD, Bridwell K, Dimar JR, Horton W, Berven S, Schwab F : The impact of positive sagittal balance in adult spinal deformity. Spine (Phila Pa 1976) 30 : 2024-2029, 2005

14. Gomez JA, Makhni MC, Vitale MG : Recurrent spinal deformity after scoliosis surgery in children. Instr Course Lect $63:$ 345-351, 2014

15. Han S, Hyun SJ, Kim KJ, Jahng TA, Lee S, Rhim SC : Rod stiffness as a risk factor of proximal junctional kyphosis after adult spinal deformity surgery: comparative study between cobalt chrome multiple-rod constructs and titanium alloy two-rod constructs. Spine J 17 : 962-968, 2017

16. Hanson DS, Bridwell KH, Rhee JM, Lenke LG : Correlation of pelvic incidence with low- and high-grade isthmic spondylolisthesis. Spine (Phila Pa 1976) 27 : 2026-2029, 2002

17. Hardacker JW, Shuford RF, Capicotto PN, Pryor PW : Radiographic standing cervical segmental alignment in adult volunteers without neck symptoms. Spine (Phila Pa 1976) 22 : 1472-1480; discussion 1480, 1997

18. Harimaya K, Lenke LG, Mishiro T, Bridwell KH, Koester LA, Sides BA : Increasing lumbar lordosis of adult spinal deformity patients via intraoperative prone positioning. Spine (Phila Pa 1976) 34 : 2406-2412, 2009

19. Harroud $A$, Labelle $H$, Joncas J, Mac-Thiong JM : Global sagittal alignment and health-related quality of life in lumbosacral spondylolisthesis. Eur Spine J 22 : 849-856, 2013

20. Hwang JH, Modi HN, Suh SW, Hong JY, Park YH, Park JH, et al. : Reliability of lumbar lordosis measurement in patients with spondylolisthesis: a case-control study comparing the Cobb, centroid, and posterior tangent methods. Spine (Phila Pa 1976) 35 : 1691-1700, 2010

21. Hyun SJ, Kim KJ, Jahng TA, Kim HJ : Relationship between T1 slope and cervical alignment following multilevel posterior cervical fusion surgery: impact of T1 slope minus cervical lordosis. Spine (Phila Pa 1976) 41 : E396-E402, 2016

22. Hyun SJ, Kim YJ, Rhim SC : Patients with proximal junctional kyphosis after stopping at thoracolumbar junction have lower muscularity, fatty degeneration at the thoracolumbar area. Spine J 16 : 1095-1101, 2016

23. Hyun SJ, Lee BH, Park JH, Kim KJ, Jahng TA, Kim HJ. Proximal junctional kyphosis and proximal junctional failure following adult spinal deformity surgery. Korean J Spine 14 : 126-132, 2017

24. Hyun SJ, Lenke LG, Kim YC, Koester LA, Blanke KM : Comparison of 
standard 2-rod constructs to multiple-rod constructs for fixation across 3-column spinal osteotomies. Spine (Phila Pa 1976) 39 : 1899-1904, 2014

25. Hyun SJ, Rhim SC : Clinical outcomes and complications after pedicle subtraction osteotomy for fixed sagittal imbalance patients : a long-term follow-up data. J Korean Neurosurg Soc 47 : 95-101, 2010

26. Iyer S, Lenke LG, Nemani VM, Fu M, Shifflett GD, Albert TJ, et al. : Variations in occipitocervical and cervicothoracic alignment parameters based on age: a prospective study of asymptomatic volunteers using full-body radiographs. Spine (Phila Pa 1976) 41 : 1837-1844, 2016

27. Kim HJ, Boachie-Adjei O, Shaffrey Cl, Schwab F, Lafage V, Bess S, et al. : Upper thoracic versus lower thoracic upper instrumented vertebrae endpoints have similar outcomes and complications in adult scoliosis. Spine (Phila Pa 1976) 39 : E795-E799, 2014

28. Kim Y, Bridwell KH, Lenke LG, Boachie-Adjei O, Hamill CL, Cho S, et al. : Sagittal decompensation following pedicle subtraction osteotomy for adult patients with sagittal imbalance: incidence, risk factors, and SRS outcomes score. Spine 10(Suppl 3) : 130, 2009

29. Kim YJ, Bridwell KH, Lenke LG, Boachie-Adjei O, Hamill C, Kim YB : Sagittal spinopelvic alignment change after lumbar pedicle subtraction osteotomy: a multicenter analysis of 113 patients with a minimum 2 years follow-up. SRS 2008: Proceedings of the 43rd annual meeting of scoliosis research society, Salt Lake City, 2008

30. Kim Y, Bridwell KH, Lenke LG, Kim YB, Kim P : Comparative radiographic analysis of the sagittal spinopelvic alignment between 100 asymptomatic adults and 100 patients with sagittal imbalance: the best angular parameters to sagittal vertical axis. Spine 10(Suppl 3) : 178-180, 2009

31. Kim YJ, Bridwell KH, Lenke LG, Cheh G, Baldus C : Results of lumbar pedicle subtraction osteotomies for fixed sagittal imbalance: a minimum 5-year follow-up study. Spine (Phila Pa 1976) 32 : 2189-2197, 2007

32. Kim YJ, Bridwell KH, Lenke LG, Rhim S, Cheh $G$ : An analysis of sagittal spinal alignment following long adult lumbar instrumentation and fusion to L5 or S1: can we predict ideal lumbar lordosis? Spine (Phila Pa 1976) 31 : 2343-2352, 2006

33. Kim YJ, Hyun SJ, Cheh G, Cho SK, Rhim SC : Decision making algorithm for adult spinal deformity surgery. J Korean Neurosurg Soc 59 : 327-333, 2016

34. Knott PT, Mardjetko SM, Techy F : The use of the T1 sagittal angle in predicting overall sagittal balance of the spine. Spine J 10 : 994-998, 2010

35. Lafage V, Ames C, Schwab F, Klineberg E, Akbarnia B, Smith J, et al. Changes in thoracic kyphosis negatively impact sagittal alignment after lumbar pedicle subtraction osteotomy: a comprehensive radiographic analysis. Spine (Phila Pa 1976) 37 : E180-E187, 2012

36. Lee CS, Chung SS, Kang KC, Park SJ, Shin SK : Normal patterns of sagittal alignment of the spine in young adults radiological analysis in a Korean population. Spine (Phila Pa 1976) 36 : E1648-E1654, 2011

37. Lee SH, Kim KT, Seo EM, Suk KS, Kwack YH, Son ES : The influence of thoracic inlet alignment on the craniocervical sagittal balance in asymptomatic adults. J Spinal Disord Tech 25 : E41-E47, 2012

38. Lee SH, Son ES, Seo EM, Suk KS, Kim KT : Factors determining cervical spine sagittal balance in asymptomatic adults: correlation with spinopel- vic balance and thoracic inlet alignment. Spine J 15 : 705-712, 2015

39. Legaye J, Duval-Beaupère G, Hecquet J, Marty C : Pelvic incidence: a fundamental pelvic parameter for three-dimensional regulation of spinal sagittal curves. Eur Spine J 7 : 99-103, 1998

40. Mac-Thiong JM, Pinel-Giroux FM, de Guise JA, Labelle H : Comparison between constrained and non-constrained Cobb techniques for the assessment of thoracic kyphosis and lumbar lordosis. Eur Spine J 16 : 13251331, 2007

41. Nunez-Pereira S, Hitzl W, Bullmann V, Meier O, Koller H : Sagittal balance of the cervical spine: an analysis of occipitocervical and spinopelvic interdependence, with C-7 slope as a marker of cervical and spinopelvic alignment. J Neurosurg Spine 23 : 16-23, 2015

42. Park JH, Hyun SJ, Kim KJ, Jahng TA : Comparative study between pedicle subtraction osteotomy (PSO) and closing-opening wedge osteotomy (fish-mouth PSO) for sagittal plane deformity correction. Spine (Phila Pa 1976) 24 : E899-E905, 2016

43. Potter BK, Lenke LG, Kuklo TR : Prevention and management of iatrogenic flatback deformity. J Bone Joint Surg Am 86-A : 1793-1808, 2004

44. Qiao J, Zhu F, Xu L, Liu Z, Zhu Z, Qian B, et al. : T1 pelvic angle: a new predictor for postoperative sagittal balance and clinical outcomes in adult scoliosis. Spine (Phila Pa 1976) 39 : 2103-2107, 2014

45. Rajnics $P$, Templier $A$, Skalli $W$, Lavaste F, Illés $T$ : The association of sagittal spinal and pelvic parameters in asymptomatic persons and patients with isthmic spondylolisthesis. J Spinal Disord Tech 15 : 24-30, 2002

46. Rose PS, Bridwell KH, Lenke LG, Cronen GA, Mulconrey DS, Buchowski JM, et al. : Role of pelvic incidence, thoracic kyphosis, and patient factors on sagittal plane correction following pedicle subtraction osteotomy. Spine (Phila Pa 1976) 34 : 785-791, 2009

47. Roussouly P, Nnadi C : Sagittal plane deformity: an overview of interpretation and management. Eur Spine J 19 : 1824-1836, 2010

48. Ryan DJ, Protopsaltis TS, Ames CP, Hostin R, Klineberg E, Mundis GM, et al. : T1 pelvic angle (TPA) effectively evaluates sagittal deformity and assesses radiographical surgical outcomes longitudinally. Spine (Phila Pa 1976) 39 : 1203-1210, 2014

49. Saville $P A$, Kadam $A B$, Smith $H E$, Arlet $V$ : Anterior hyperlordotic cages: early experience and radiographic results. J Neurosurg Spine 25 : 713719,2016

50. Schwab F, Dubey A, Gamez L, El Fegoun AB, Hwang K, Pagala M, et al. : Adult scoliosis: prevalence, SF-36, and nutritional parameters in an elderly volunteer population. Spine (Phila Pa 1976) 30 : 1082-1085, 2005

51. Schwab F, Lafage V, Patel A, Farcy JP : Sagittal plane considerations and the pelvis in the adult patient. Spine (Phila Pa 1976) 34 : 1828-1833, 2009

52. Schwab F, Patel A, Ungar B, Farcy JP, Lafage V : Adult spinal deformitypostoperative standing imbalance: how much can you tolerate? An overview of key parameters in assessing alignment and planning corrective surgery. Spine (Phila Pa 1976) 35 : 2224-2231, 2010

53. Suk SI, Kim JH, Kim WJ, Lee SM, Chung ER, Nah KH : Posterior vertebral column resection for severe spinal deformities. Spine (Phila Pa 1976) $27: 2374-2382,2002$

54. Thomasen $\mathrm{E}$ : Vertebral osteotomy for correction of kyphosis in ankylos- 
Restoring Sagittal Balance | Makhni MC, et al.

ing spondylitis. Clin Orthop Relat Res (194) : 142-152, 1985

55. Vialle R, Levassor N, Rillardon L, Templier A, Skalli W, Guigui P : Radiographic analysis of the sagittal alignment and balance of the spine in asymptomatic subjects. J Bone Joint Surg Am 87 : 260-267, 2005
56. Wang $Y$, Zhang $Y$, Zhang $X$, Wang Z, Mao K, Chen C, et al. : Posterioronly multilevel modified vertebral column resection for extremely severe Pott's kyphotic deformity. Eur Spine J 18 : 1436-1441, 2009 